\title{
APLICABILIDAD DE LA FIGURA DEL DELITO CONTINUADO EN LOS DELITOS SEXUALES. COMENTARIO A UN FALLO
}

\author{
GUILlermo Oliver CALDERÓN* \\ LUIS RODRÍGUEZ COLLAO**
}

\section{INTRODUCCIÓN}

En una sentencia de fecha 24 de diciembre de 2008, dictada en la causa RIT 228-2008 (RUC 0810002040-9), sobre supuestos delitos de significación sexual, la Primera Sala del Tribunal de Juicio Oral en lo Penal de Viña del Mar declaró que en los hechos establecidos en el fallo no correspondía apreciar un delito continuado y que la aplicación de esta figura no resulta procedente cuando se trata de delitos sexuales.

El objetivo de este trabajo es analizar críticamente ambas afirmaciones hechas por el tribunal. Tangencialmente, se abordarán también otros aspectos de la sentencia que merecen una opinión, especialmente en lo relativo al procedimiento utilizado en la individualización de la pena. Los párrafos pertinentes del fallo han sido transcritos en forma literal, salvo en aquella parte en que se contiene la identidad del imputado y de otras personas, que hemos decidido omitir para mantener a salvo su dignidad.

\section{1) SÍNTESIS DE LA SENTENCIA}

Conforme a lo expresado en el considerando undécimo del citado fallo, los hechos que el tribunal dio por establecidos fueron los siguientes:

"En el mes de febrero del año 2006, el acusado A, a la época de 20 años de edad, llegó hasta Peńablanca, al domicilio de B, nacido el 4 de junio de 1992, y a la sazón de 13 ańos de edad, en calidad de amigo del hermano mayor de la víctima, C. Pasada la medianoche, aprovechando que la madre de la víctima no se encontraba en el inmueble, el padrastro

\footnotetext{
3. Doctor en Derecho, U. de Barcelona, Profesor adjunto de Derecho Penal y Derecho Procesal Penal. Pontificia U. Católica de Valparaíso. Correo electrónico: guillermo.oliver@ucv.cl

* Doctor en Derecho, U. de Lérida, Profesor titular de Derecho Penal, Pontificia U.

Católica de Valparaíso. Correo electrónico: lrodrigc@ucv.cl

Fecha de recepción: 9 de abril de 2009.

Fecha de aprobación: 4 de junio de 2009.
} 
de este dormía y su hermano mayor C se había ido a acostar, en los momentos en que jugaban play station en el dormitorio de la víctima, el imputado comenzó a tocarle el cuerpo y la zona genital, intentando resistirse el menor, fue empujado sobre la cama sacándole sus pantalones y slips, penetrándolo con su pene por el ano. Acto seguido, obligó a la víctima a penetrarlo por el ano, luego de lo cual le exigió que se quedara callado y no le contara a nadie, pernoctando esa noche en el domicilio. A la mañana siguiente, el imputado, no obstante la negativa de la víctima volvió a penetrarla con su pene por el ano, aprovechando que se encontraban solos en una pieza destinada a oficina en la casa, bajándole los pantalones y ropa interior, nuevamente advirtiéndole que no contara nada a nadie y que sería la última vez.

Desde el mes de marzo del 2006, el imputado concurrió con frecuencia al nuevo domicilio de B y su familia, ubicado en Villa Alemana, donde aprovechaba los momentos en que se encontraba solo, repitiendo los episodios en que penetraba con su pene por el ano a la víctima con una frecuencia semanal, siendo la última ocasión la ocurrida el día 23 de enero del 2008. Finalmente el día sábado 26 de enero del 2008, la víctima develó todo a su hermana $\mathrm{D}$, quien a su vez lo transmitió al resto de la familia, interponiendo la correspondiente denuncia en la Sexta Comisaría de Carabineros de Villa Alemana, lugar al cual había ingresado previamente el acusado, siendo detenido posteriormente por estos hechos".

Estos hechos fueron calificados por el tribunal como constitutivos de dos reiteraciones, una de delitos de violación impropia y otra de delitos de sodomía, como se desprende del considerando duodécimo de la mencionada sentencia, cuyo tenor, en su parte pertinente, es el siguiente:

"Que los hechos consignados en el razonamiento anterior, constituyeron para el Tribunal la calificación jurídica de los mismos dentro de las figuras de los delitos de violación contemplado en el artículo 362 y de sodomía, establecido en el art. 365, ambos del Código Penal, en calidad de reiterados y cometidos en grado de consumados, toda vez que el hechor, en diversas oportunidades, accedió carnalmente, vía anal, a un joven de su mismo sexo, desde que la víctima contaba con 13 años de edad, hasta casi dos ańos después de ocurrido el primer evento.

En consecuencia, se desestimaron los cargos de la Fiscalía en cuanto a tener por establecido el delito de estupro (art. 363), el que requiere para su configuración - de la hipótesis del numeral 4- que el hechor haya engańado a la víctima, y además que ese engaño se efectúe abusando de su inexperiencia o ignorancia sexual, los que dados los reiterados episodios de relaciones sexuales, anteriores al 4 de junio de 2006 (fecha en que B cumplió 14 ańos de edad) obsta a sostener que el afectado carecía de experiencia sexual o de conocimientos mínimos de sexualidad, si por ello entendemos como "experiencia", el "hecho de haber sentido, conocido o 
presenciado alguien algo" (Diccionario de la Real Academia Española), y en especial, no es posible concluir que en el menor hubiese existido "una falta de conocimiento derivada de no haber ejercitado una persona actividad sexual o de los hechos de su propia vida, que pudieron mantenerla alejada de la información que el común de las personas adquiere acerca de la sexualidad humana" (Delitos sexuales, Luis Rodríguez Collao, pág. 180), pues desde el mes de febrero de 2006, con una frecuencia semanal, B era partícipe de actividad sexual con el imputado".

El tribunal desechó la aplicación a estos hechos de la figura del delito continuado, por las razones esgrimidas en el considerando decimoséptimo, que se transcribe a continuación:

"Sobre el delito continuado, se descarta su aplicación en este tipo de ilícitos debiendo apreciarse en todos los casos como un delito reiterado, ya que el bien jurídico cautelado consistente en la libertad sexual individual, es uno personalísimo, que se vulnera con cada ataque de que es objeto, puesto que el tipo se satisface en todas las oportunidades en que el hechor, en un distinto contexto situacional, accede carnalmente al sujeto pasivo" (Manual de Delitos Sexuales. Cristián Aguilar Aranela, pág. 37). En tal sentido, la jurisprudencia ha coincidido pudiendo citarse al efecto la causa Rol 649-2004 (de 12/10/2004) donde la Corte de Apelaciones de Valparaíso concluyó que "hay determinados bienes jurídicos respecto de los que no cabe invocar la figura del delito continuado, dentro de ellos, la libertad sexual, la vida y otros, en que la acción dirigida a atacar bienes de esa clase, junto con satisfacer el tipo respectivo lo colman, por lo que estamos en presencia aquí de una reiteración de delitos, esto es, de diversos actos, separados cronológicamente el uno del otro, de modo que el hechor puede ser castigado por cada uno de ellos aisladamente" (Corte de Apelaciones de Antofagasta, 02/01/2006, Rol 275-2005 RPP y 18/01/2006, Rol 284-2005. Tribunales de Juicio Oral en lo Penal de Iquique, Viña del Mar, Punta Arenas, Curicó, Calama y Antofagasta, y de Garantía de Antofagasta, con fechas 15/7/2004; 29/10/2004; 01/06/2004; 26/6/2004; $17 / 6 / 2004$ y $30 / 4 / 2005$; y 29/12/2005, en causas RUC 0300106319-6, 0300211125-9, 0300133399-1, 0300029387- 2 y 0200011127-1; RIT 41-2005 y RUC 05001 62087-K, respectivamente".

En lo que respecta a las penas principales impuestas, el tribunal, como se desprende del considerando vigésimo segundo del fallo, ha condenado al imputado a diez años y un día de presidio mayor en su grado medio, como autor de violaciones reiteradas, y a quinientos cuarenta y un días de reclusión menor en su grado medio, como autor de delitos reiterados de sodomía. El procedimiento seguido por el tribunal en la individualización de la pena aparece explicitado en el considerando vigésimo primero de la sentencia, cuyo tenor es el siguiente:

"Que para la determinación de la sanción legal, en la situación en estudio, se tendrá presente lo siguiente: 
(a) La pena asignada por la ley para el delito de violación es la de presidio mayor en cualquiera de sus grados; y respecto de la sodomía es de reclusión menor en sus grados mínimo a medio.

(b) Que ambos delitos se encuentran consumados y reiterados, por lo que de acuerdo a lo establecido en el artículo 351 del Código Procesal Penal, las diversas infracciones se considerarán respectivamente, como un solo delito, aumentándose la pena correspondiente en un grado, con lo cual se llega respecto de la violación, a presidio mayor en su grado medio y en la sodomía, a reclusión menor en su grado medio.

(c) Que en cada uno de los delitos referidos, favorece al acusado una atenuante y no le perjudican agravantes, y en tales circunstancias el artículo 67 del Código Penal dispone que la pena ha de aplicarse en su mínimum, es decir, en su mitad inferior.

(d) Que por ser más favorable al condenado, se le impondrán todas las penas correspondientes a las diversas infracciones (art. 74 del Código Penal), debiendo el sentenciado cumplir todas sus condenas simultáneamente, principiando por la más grave, esto es la correspondiente al delito de violación".

\section{2) Comentario}

\section{(2.1) Pluralidad DE CONDUCTAS DELictivas EN Distintos CON- TEXTOS SITUACIONALES: EL DELITO CONTINUADO}

Puede definirse el delito continuado como aquel constituido por dos o más acciones u omisiones separadas por un cierto tiempo que, no obstante integrar cada una de ellas por separado la misma figura fundamental de delito, se valoran como uno solo en razón de la homogeneidad de sus elementos. Ningún precepto legal se refiere en nuestro país a esta clase de delito, sin embargo puede decirse que goza de considerable reconocimiento en la jurisprudencia y doctrina nacionales. Su origen se encuentra en los prácticos italianos, que crearon este instituto para evitar la aplicación de una disposición legal muy severa que sancionaba con la pena de muerte a la horca a quienes incurrían en tres o más hurtos. Con posterioridad fue recogido en la legislación italiana, y de allí se trasladó a la dogmática extranjera, que generalmente lo ha elaborado sin contar con legislación positiva que lo consagre.

Suele señalarse que para estar en presencia de un delito continuado deben concurrir una serie de requisitos, tanto objetivos como subjetivos. Del primer orden son: la pluralidad de acciones u omisiones, la unidad de ley violada y, para algunos, la identidad del sujeto pasivo. De índole subjetiva es el requisito de unidad de designio, propósito, intención o dolo. 
También se incluyen en la doctrina ciertos elementos de carácter secundario que, fundamentalmente, permiten dar por establecido alguno de los requisitos mencionados. Aquí se ubican la unidad o identidad de ocasión, la conexión espacial y temporal, y el empleo de medios semejantes.

\section{(2.1.1) Elementos objetivos}

(a) Pluralidad de acciones u omisiones. Lo que da lugar al delito continuado es que las diversas acciones ejecutadas son, cada una de ellas, típicas. Por esa misma razón, debe darse un cierto espaciamiento temporal entre ellas.

(b) Realización del mismo tipo básico. También es indispensable que las diversas acciones realizadas vulneren unos mismos o semejantes preceptos legales. No se requiere, entonces, que todas las conductas merezcan idéntica calificación; basta con que las diversas figuras sean análogas, esto es, que puedan ser comprendidas como manifestaciones de un tipo básico. Luego podrá darse tal homogeneidad, por ejemplo, entre un hurto y un robo.

(c) Identidad de sujeto pasivo. No todos los autores concuerdan en esta exigencia. Tiende a uniformarse la doctrina en torno a la idea de que ella solo es importante en los delitos que protegen bienes personalísimos, tales como la vida, la salud, la libertad (ambulatoria y sexual) y el honor, pero no así en los delitos de significación patrimonial.

\section{(2.1.2) Elemento subjetivo}

Sin duda este es el elemento más controvertido. Paralelamente a los caracteres objetivos que denotan una cierta unidad en las acciones realizadas, se exige también un factor aglutinante de índole subjetiva. Para muchos, es este el factor decisivo y, en todo caso, el que ha de justificar el tratamiento más benigno que se persigue con la figura del delito continuado.

Las múltiples posturas adoptadas al respecto pueden reducirse a dos: las que reclaman una unidad del elemento subjetivo, sea que se lo conciba como dolo, resolución, propósito o deseo, y las que exigen una cierta continuidad u homogeneidad entre las diversas resoluciones o dolos. La primera alternativa, esto es, la que postula la unidad del dolo, propósito o intención, tiene el grave inconveniente de que si el dolo es unitario, ha de serlo por abarcar de antemano todas las acciones parciales en un propósito común. Pero si es así, esa misma perseverancia en la intención delictiva, lejos de disminuir la reprochabilidad del hecho, la aumenta, lo cual no concuerda con la búsqueda de un tratamiento más benigno para el imputado, a través de la consideración unitaria de sus acciones para evitar la aplicación de las reglas agravatorias del concurso real de delitos. Téngase presente que por algo el Código Penal considera como agravante 
la premeditación conocida en los delitos contra las personas (artículo 12 No 5).

Más congruente se revela la segunda postura, que prefiere poner el acento en la homogeneidad del dolo del agente, la cual vendría dada por obedecer todas las resoluciones delictivas a circunstancias externas semejantes. Así, podrá afirmarse la existencia de un delito continuado cuando el individuo haya actuado sucumbiendo en múltiples ocasiones a la tentación provocada por las mismas o semejantes circunstancias motivantes a la comisión delictiva. Al mismo tiempo, será posible afirmar que su reprochabilidad es menor en la medida en que sus actos revelan una voluntad débil, con lo que el tratamiento unitario y favorable de las diversas acciones adquiere justificación. Es esta la tesis dominante en la doctrina alemana.

En nuestro país, Etcheberry se pronuncia por la unidad de propósito o determinación. Garrido Montt adhiere a ambas concepciones del elemento subjetivo del delito continuado, esto es, tanto a la de la unidad como a la de la continuidad u homogeneidad del dolo. A nuestro parecer, la posición correcta es la que atiende únicamente a la semejanza de las circunstancias motivantes del comportamiento del individuo, por ser la única que puede dar sustento a un tratamiento unitario y más favorable a las diversas acciones delictivas ejecutadas por él. Siendo posible recurrir a esta construcción doctrinal solo en beneficio del imputado, su justificación ha de encontrarse en una disposición subjetiva que trasunte una menor reprochabilidad, y no a la inversa.

En cuanto a su tratamiento penológico, la lógica consecuencia de considerar que el delito continuado es uno solo, es la de aplicarle la pena correspondiente al hecho único cometido. De este modo, si son varias las conductas constitutivas de hurto o de apropiación indebida, por ejemplo, cabría simplemente sumar el importe total de lo sustraído o del perjuicio, en su caso, e imponer la pena resultante. La sanción para el conjunto de acciones pasa a ser idéntica, entonces, a la que habría sido aplicable si el hecho se hubiera ejecutado por medio de una sola acción. Esto, en verdad, resulta fácilmente practicable cuando las conductas realizadas se castigan con penas que se establecen sobre una base acumulable. Tal cosa ocurre en los casos citados, pues en ellos la pena se calcula sobre la base de los valores económicos involucrados. Pero en los demás casos, esto es, cuando las penas no admiten integración en una sola, la búsqueda de una penalidad única para todas las infracciones conduce a optar por la pena aplicable a una de ellas, concretamente, la correspondiente a la infracción más grave.

\section{(2.2) Procedencia de la APLICACión DE la Figura DEL DELITO CONTINUADO EN LOS DELITOS SEXUALES}

Tal como lo hemos sostenido, la figura del delito continuado, si bien no está consagrada en el derecho positivo chileno, es ampliamente aco- 
gida tanto por la doctrina científica como por la jurisprudencial. Pese a que normalmente se plantea que esta figura, cuya elaboración primitiva se vincula con los delitos patrimoniales, ha ido ampliando su campo de aplicación, la verdad es que la doctrina siempre ha manifestado una opinión contraria a su admisibilidad en el campo de los delitos que atentan en contra de bienes jurídicos de índole estrictamente personal; y este planteamiento, por lo general, se hace extensivo a los delitos sexuales.

Acogiendo la posición mayoritaria, el Código Penal español de 1995, que en su artículo 74 regula expresamente la figura del delito continuado, la declaró inadmisible respecto de "las ofensas a bienes eminentemente personales", pero permitió aplicarla en los delitos contra el honor y la libertad sexual. Pese a lo categórico de esta declaración, un sector de la doctrina española sigue sosteniendo la imposibilidad de vincular esta figura con las infracciones de carácter sexual.

Recapitulando lo dicho más arriba acerca del concepto y de los requisitos del delito continuado, suele entenderse por tal una pluralidad de conductas ejecutadas en tiempos distintos, cada una de las cuales reúne los requisitos necesarios para ser considerada como delito independiente, pero que presentan ciertos rasgos comunes, tanto en el plano objetivo (unidad de sujeto pasivo y de bien jurídico lesionado), como desde un punto de vista subjetivo (unidad de resolución delictiva). Bajo estas condiciones, y al menos desde un punto de vista conceptual, no vemos inconveniente para admitir la aplicabilidad de esta figura respecto de la generalidad de los delitos sexuales; si bien reconocemos que las situaciones en que ello puede ocurrir son excepcionales. Una situación imaginable en que ello podría suceder sería la del sujeto que atenta en varias oportunidades en contra de una misma persona, por ejemplo, a título de violación, estupro o abuso sexual, aprovechando, durante toda la secuencia delictual, la persistencia de una misma situación de desvalimiento de la víctima.

Con todo, es preciso tener en consideración tres cosas. Primero, que la figura del delito continuado tiene su razón de ser en el propósito de no agravar la situación penal del individuo que ejecuta varias conductas en el marco de una misma resolución delictiva, de manera que la pena aplicable por este concepto, nunca puede ser superior a la que resulte de considerar en forma aislada esas mismas conductas. Segundo, que el delito continuado supone que las varias acciones sean ejecutadas en tiempos u ocasiones distintos, de manera que la repetición de una misma conducta dentro de un mismo contexto situacional no es un caso de continuidad delictiva, sino un ejemplo de unidad de acción, que da lugar a la configuración de un solo delito. Y, tercero, que varios tipos admiten e incluso algunos exigen la realización plural de un mismo comportamiento, como sucede en el caso de la figura agravada de favorecimiento de la prostitución de menores del inciso segundo del artículo 367 del Código Penal. 


\section{(2.3) APLICABILIDAD DEL DELITO CONTINUADO EN LOS HECHOS ES- TABLECIDOS}

Conforme a lo anterior, es posible advertir con relativa facilidad que en los hechos que el tribunal dio por establecidos sí resultaba procedente la aplicación de la figura del delito continuado, ya que todos y cada uno de sus requisitos efectivamente concurrían. Por lo que respecta a la pluralidad de acciones u omisiones espaciadas temporalmente, esta se da por establecida en el considerando undécimo del fallo, que alude a una penetración anal pasada la medianoche de un día de febrero de 2006, otra que tuvo lugar a la mańana siguiente y otras más entre los meses de marzo de 2006 y enero del 2008. En cuanto a la exigencia de que las varias acciones vulneren un mismo artículo o preceptos legales semejantes, este requisito también concurre. En efecto, hasta antes del 4 de junio de 2006 -fecha en la que la víctima cumplió catorce ańos- las penetraciones anales implicaron realizar el tipo de violación impropia del artículo 362 del Código Penal, y las posteriores, el tipo de sodomía -o corrupción de menoresdel artículo 365 del mismo cuerpo normativo. Ambas figuras pueden ser consideradas homogéneas o análogas, en tanto atentan contra el mismo bien jurídico, el derecho a la indemnidad sexual.

La exigencia de identidad de sujeto pasivo también se cumple. Conforme a los hechos establecidos por el tribunal en su sentencia, las diversas acciones sexuales tuvieron como víctima a una misma persona. Finalmente, el elemento subjetivo también concurre. Como se ha expresado más arriba, la manera más congruente de concebirlo es la que pone el acento en la homogeneidad del dolo del sujeto, la que vendría dada por obedecer todas las resoluciones delictivas a circunstancias externas semejantes. De este modo, el agente habría actuado sucumbiendo en varias ocasiones a la tentación provocada por las mismas o semejantes circunstancias motivantes a la comisión delictiva. Pues bien, es el propio tribunal el que, en los hechos establecidos, implícitamente ha dado por concurrente este elemento subjetivo. Así se desprende de la parte pertinente del citado considerando undécimo, en el que se expresa que la primera acción sexual fue realizada por el imputado, "aprovechando que la madre de la víctima no se encontraba en el inmueble, el padrastro de esta dormía y su hermano mayor C se había ido a acostar", que la segunda fue realizada "aprovechando que se encontraban solos", y que las restantes tuvieron lugar cuando el imputado "aprovechaba los momentos en que se encontraba solo". Es decir, fueron las mismas o semejantes circunstancias externas -haberse encontrado sola la víctima- las que motivaron las resoluciones delictivas, sucumbiendo a la tentación provocada por aquellas.

Como puede apreciarse, todos los requisitos del delito continuado han concurrido, razón por la cual se imponía su aplicación en la calificación jurídica de los hechos establecidos por el tribunal. Y en cuanto a la 
manera de sancionar esta continuidad delictiva, tal como lo hemos sostenido más arriba, debería haberse considerado la pena correspondiente a la acción más grave, es decir, la pena de un único delito de violación impropia del artículo 362 del Código Penal.

A lo anterior debe agregarse que existe otra razón para concluir que solo debió haberse condenado por delito continuado de violación impropia, que toma en cuenta consideraciones valorativas. El fundamento del castigo del delito de sodomía del artículo 365 del Código Penal es el atentado contra la indemnidad sexual, por el efecto corruptivo que la acción allí descrita puede traer consigo para el mayor de catorce pero menor de dieciocho ańos. Pues bien, no debe olvidarse que ese es exactamente el mismo fundamento que sirve para justificar la mayor pena que el Código Penal asigna a la conducta de acceder carnalmente a un menor de catorce ańos, conforme al artículo 362 del mencionado Código. Por lo tanto, castigar a título de violación impropia y a título de sodomía, como se ha hecho en el fallo analizado, atenta contra el principio non bis in idem. En otras palabras, imponer pena por delito de sodomía importa una vulneración de la prohibición de doble valoración, ya que implica sancionar por algo que ya ha sido considerado al imponer la pena más grave que se contempla para el delito de violación impropia.

Por otra parte, la lectura de la sentencia examinada invita a formularse la siguiente pregunta: ¿Qué habría hecho el tribunal si los diversos ataques sexuales no hubieran tenido como víctima a una misma persona, sino a distintas personas? Seguramente habría hecho lo mismo, esto es, apreciar una reiteración de delitos de la misma especie. Esto demuestra la incorrección del procedimiento seguido en la determinación del título de castigo. No sería justo adoptar la misma solución en ambos casos. En uno -que corresponde a los hechos establecidos en el fallo- se ha afectado la indemnidad sexual de solo una persona; en tanto que en el otro, se habría atentado contra la indemnidad sexual de varias personas. Aplicando las figuras del delito continuado para el primer caso y de la reiteración para el segundo, se respetaría la justicia comparativa.

A las razones seńaladas cabe agregar otra para concluir que debió haberse aplicado la figura del delito continuado a los hechos establecidos por el tribunal. Se trata de lo que Etcheberry denomina indeterminación procesal. Consiste en la evidente tendencia jurisprudencial a aplicar esta figura cuando no resulta posible precisar el número exacto de acciones delictivas imputadas ni las fechas precisas de comisión. Existen varias sentencias de tribunales superiores del país en este sentido.

Según nuestra opinión, existen buenos argumentos para sostener que respecto de varias acciones $\mathrm{u}$ omisiones típicas se origina esta indeterminación procesal, con mayor razón debe aplicarse la figura del delito continuado. Cuando la imputación de varios hechos delictivos formulada en contra de una persona se hace en forma indeterminada, sin indicar su número 
exacto ni las fechas precisas en que habrían tenido lugar (por ejemplo: “...el sujeto X, en varias ocasiones, entre el 10 de enero de 2007 y el 10 de enero de 2008, ejecutó los hechos...”), resulta evidente que se produce alguna afectación en su derecho de defensa. Es mucho más difícil defenderse de una imputación así de indeterminada que de otra que señale con precisión el número de hechos y las fechas en que habrían sucedido. Ello, porque ante una imputación de esta última clase, bastaría para desvirtuarla con acreditar que en las concretas fechas en que los hechos habrían acaecido el imputado estaba en un lugar distinto al de la comisión del delito, o que la víctima se encontraba en otro lugar, o cualquier otra circunstancia que hiciera imposible su comisión por parte del imputado. Pero ante una imputación indeterminada en las fechas y número de los hechos, la labor de la defensa se dificulta, ya que para desvirtuarla habría que probar dicha imposibilidad de comisión durante un período prolongado. Pues bien, como una forma de compensar esta evidente perturbación en la tarea de la defensa, parece razonable sostener la aplicabilidad de la figura del delito continuado en estas hipótesis de indeterminación procesal.

Además, existe una razón formal que avala este planteamiento, cual es, el modo en que está redactado el artículo 351 del Código Procesal Penal. Esta disposición, que establece la manera como se sanciona la reiteración de delitos de la misma especie, señala en su inciso segundo que cuando por la naturaleza de las diversas infracciones estas no pudieren ser estimadas como un solo delito ${ }^{1}$, debe aplicarse la pena que corresponda al delito que tenga, en el caso concreto, una pena mayor, y aumentarla en uno o dos grados "según fuere el número de los delitos". Esto es una demostración de que para poder aplicar la regla de punición de la reiteración de delitos, es necesario saber cuál es el número de delitos que se atribuyen al imputado. La mencionada regla opera sobre la base de que se tenga certeza acerca del número de delitos de que se trate. Corrobora esta idea el hecho de que el inciso penúltimo de la citada disposición contiene una remisión a la regla general de castigo del concurso material de delitos del artículo 74 del Código Penal, regla de la acumulación aritmética que debe ser aplicada si así se llega a una pena menor. Pues bien, la única manera posible de saber si la regla del artículo 74 del Código Penal conduce a una pena menor, es teniendo claro el número de delitos imputados. Si se aplicara la regla de la reiteración de delitos de la misma especie del artículo 351 del Código Procesal Penal a las hipótesis de indeterminación procesal en lo que respecta al número de delitos, lo dispuesto en su inciso penúltimo nunca podría aplicarse, convirtiéndose en letra muerta.

Lo cual, según la doctrina mayoritaria, significa que las penas de los distintos delitos no se encuentran estructuradas sobre bases acumulables que se puedan sumar, situación que es precisamente la de los hechos establecidos por el tribunal. 


\section{(2.4) OTROS MOTIVOS DE ERRÓNEA APLICACIÓN DEL DERECHO EN EL FALLO ANALIZADO. DEFECTUOSA INDIVIDUALIZACIÓN DE LA PENA IMPUESTA}

Según nuestra opinión, además de haberse efectuado una errónea aplicación del Derecho por parte del tribunal al haber calificado los hechos establecidos como una reiteración de delitos y no como un delito continuado, se ha incurrido, concretamente, en el procedimiento utilizado en la individualización de la pena.

Como se desprende de la lectura del considerando vigésimo primero del fallo analizado, se ha incurrido en tres errores en la determinación de la pena impuesta, a saber:

(a) Como primer paso en la individualización de la pena, se ha aplicado el artículo 351 del Código Procesal Penal en dos ocasiones: una, para determinar el marco penal que correspondería a las violaciones impropias reiteradas y otra, para fijar el marco penal de los delitos reiterados de sodomía, determinando así en forma separada dos marcos de pena para los distintos títulos de castigo, lo que es un error.

El supuesto de procedencia del citado artículo 351 es que se trate de una reiteración de delitos de la misma especie, entendiendo por tales los que afectan a un mismo bien jurídico. La violación impropia y la sodomía atacan al mismo bien jurídico, el derecho a la indemnidad sexual, razón por la cual todos los delitos de violación impropia y de sodomía supuestamente cometidos deberían haberse incluido en una misma y única operación de determinación de pena. En otras palabras, se debería haber aplicado en una sola ocasión el indicado artículo 351, para fijar así un único marco penal -no dos-y seguir adelante con los pasos posteriores del procedimiento de individualización de la pena.

(b) Como segundo paso en la determinación de la pena a imponer, se ha considerado a las diversas infracciones como un solo delito, aplicándose lo dispuesto en el inciso primero del citado artículo 351, lo que también es un error. Como ya se ha dicho, la posibilidad de que los diversos delitos se estimen como uno solo depende de que sus penas se encuentren establecidas sobre bases acumulables, lo que sucede por ejemplo en los hurtos, las apropiaciones indebidas, las estafas, etc. Pues bien, nada de esto ocurre en los hechos materia de este informe, razón por la cual debería haberse seguido el camino que prevé el inciso segundo de la mencionada disposición, es decir, aplicar la pena del hecho que, con sus circunstancias, tuviere penalidad mayor -en este caso, la pena de una de las violaciones impropias- y aumentarla en uno o dos grados.

(c) Como tercer y último paso en el procedimiento de individualización de la pena, el tribunal ha decidido, observando lo dispuesto en 
el inciso tercero del mencionado artículo 351, aplicar la regla de la acumulación aritmética de penas del artículo 74 del Código Penal, por conducir a una pena más favorable al condenado, lo que es otro error. Como también lo hemos señalado, la única forma de saber si efectivamente de la aplicación del citado artículo 74 hubiere de corresponder al imputado una pena menor, es conociendo el número exacto de delitos que se le atribuyen. Como en los hechos materia de este comentario se está en presencia de lo que hemos designado, siguiendo a Etcheberry, como indeterminación procesal -en cuanto al número y a las fechas de comisión de los delitos- esta operación es imposible de realizar. Pero en honor a la verdad, esta defectuosa aplicación del Derecho no es un nuevo error, sino solo una consecuencia de la desacertada decisión de haber calificado como reiteración de delitos de la misma especie una clara hipótesis de delito continuado.

\section{CONCLUSIONES}

Al finalizar este comentario, hemos decidido dejar establecidas, a modo de conclusiones, las principales afirmaciones efectuadas a lo largo del mismo.

(1) El elemento decisivo y que justifica el tratamiento más benigno que se persigue con la figura del delito continuado, suponiendo que concurran sus restantes requisitos, es el subjetivo, en la concepción que pone el acento en la homogeneidad del dolo del agente, al obedecer todas las resoluciones delictivas a circunstancias externas semejantes y sucumbir el individuo en múltiples ocasiones a la tentación provocada por su concurrencia.

(2) La pena que corresponde imponer al delito continuado, como consecuencia de considerar que es uno solo, es la correspondiente al hecho único cometido. Pero cuando las penas de las diversas infracciones no . admiten integración en una sola, se debe optar por la pena aplicable a una de ellas, concretamente, la correspondiente a la infracción más grave.

(3) No existe inconveniente en admitir la aplicabilidad del delito continuado respecto de la generalidad de los delitos sexuales, siempre que concurran sus requisitos objetivos y subjetivos, aunque es menester reconocer que las situaciones en que ello puede ocurrir son excepcionales.

(4) Todos los requisitos del delito continuado han concurrido en los hechos establecidos por el tribunal, razón por la cual se imponía su aplicación en la calificación jurídica. Y en cuanto a la manera de sancionar esta continuidad delictiva, debería haberse considerado la pena correspondiente a la acción más grave, es decir, la pena de 
un único delito de violación impropia del artículo 362 del Código Penal. Al no hacerse esto, ha tenido lugar una errónea aplicación del derecho con influencia sustancial en lo dispositivo del fallo.

(5) La calificación de delito continuado resultaba obligada, además, por la indeterminación procesal en el número exacto de infracciones imputadas y en las fechas precisas de comisión. La tendencia jurisprudencial a aplicar esta figura en los casos de indeterminación procesal es razonable, como una manera de compensar la evidente perturbación en la tarea de la defensa que supone tratar de desvirtuar una imputación abierta en las fechas y en el número de los hechos. Además, la propia redacción del artículo 351 del Código Procesal Penal sugiere que para poder apreciar una reiteración de delitos de la misma especie debe existir claridad acerca del número de delitos que se atribuyen al imputado.

(6) En el procedimiento de individualización de la pena impuesta ha tenido lugar otra errónea aplicación del Derecho, al fijar dos marcos de pena separados para los títulos de castigo de violaciones impropias reiteradas y sodomías reiteradas en la aplicación del artículo 351 del Código Procesal Penal; al aplicar lo dispuesto en el inciso primero del citado artículo 351, en vez de lo previsto en su inciso segundo, y al aplicar la regla de la acumulación aritmética del artículo 74 del Código Penal, observando lo dispuesto en el inciso tercero del citado artículo 351, en circunstancias que se estaba en presencia de una indeterminación procesal en cuanto al número y a la fecha de comisión de los hechos imputados.

\section{BIBLIOGRAFÍA}

Antón Oneca, José (1986). Derecho Penal, 2a edición anotada y puesta al día por José Julián Hernández Gujjarro y Luis Beneytez Merino, Madrid: Akal.

Arroyo de las Heras, Alfonso (1985). Manual de Derecho Penal. El Delito, tomo II, Pamplona: Aranzadi.

Cantarero Bandres, Rocío (1990). Problemas penales y procesales del delito continuado, Barcelona: PPU.

Castińeira Palou, María Teresa (1977). El delito continuado, Barcelona: Bosch.

Cerezo Mir, José (2008). Derecho Penal. Parte General, Montevideo, Buenos Aires: B de F.

Córdoba Roda, Juan; Rodríguez Mourullo, Gonzalo (1972). Comentarios al Código Penal, tomo II, Barcelona: Ariel.

Cury Urzúa, Enrique (2005). Derecho Penal. Parte General, $7^{a}$ edición, Santiago. Ediciones Universidad Católica de Chile. 
Etcheberry, Alfredo (1999). Derecho Penal. Parte General, tomo II, $3^{\text {a }}$ edición, reimpresión Santiago: Editorial Jurídica de Chile.

Etcheberry, Alfredo (1987). El Derecho penal en la jurisprudencia, tomo II, Santiago: Editorial Jurídica de Chile.

Garrido Montt, Mario (2001). Derecho Penal. Parte General, tomo II, $2^{\text {a }}$ edición, Santiago: Editorial Jurídica de Chile.

Labatut Glena, Gustavo (2000). Derecho Penal, tomo I, 9a edición actualizada por Julio Zenteno Vargas, Santiago: Editorial Jurídica de Chile.

Matus Acuña, Jean Pierre (2002), "Artículos 74 a 78", en Politoff Lifschitz, Sergio; Ortiz Quiroga, Luis (directores), Matus AcuNAa, Jean Pierre (coordinador). Texto y comentario del Código Penal chileno, tomo I, Santiago: Editorial Jurídica de Chile, pp. 383-407.

Mercado Gómez, Marco (2003). Problemas concursales y delito continuado en los delitos que protegen la libertad sexual, Santiago: LexisNexis.

Mir Puig, Santiago (2004). Derecho Penal. Parte General, $7^{\text {a }}$ edición, Montevideo - Buenos Aires: B de F.

Muñoz Conde, Francisco (2007). Derecho Penal. Parte Especial, 16a edición, Valencia: Tirant lo Blanch.

Nino, Carlos Santiago (1972). El concurso en el derecho penal. Criterios para clasificar los casos de varios hechos o de varias normas en la calificación penal de una conducta, Buenos Aires: Astrea.

Novoa Monreal, Eduardo (1985). Curso de Derecho penal chileno, tomo II, $2^{\text {a }}$ edición, Santiago: Ediar-ConoSur.

Orts Berenguer, Enrique (1996). "Delitos contra la libertad sexual" en Vives Antón, Tomás Salvador (coord.), Comentarios al Código Penal de 1995, Tirant lo Blanch: Valencia.

Politoff Lifschitz, Sergio; Matus Acuña, Jean Pierre; Ramírez Guzmán, María Cecilia (2004). Lecciones de Derecho Penal Chileno. Parte General, $1^{\text {a }}$ edición, Santiago: Editorial Jurídica de Chile.

Rodríguez Collao, Luis (2000). Delitos sexuales, Santiago: Editorial Jurídica de Chile.

Sáinz Cantero, José A. (1990). Lecciones de Derecho Penal. Parte General, $3^{\text {a }}$ edición, Barcelona: Bosch. 